\begin{tabular}{|c|c|}
\hline Title & The Noetherian Properties of the Rings of Differential Operators on Central 2-A rrangements \\
\hline Author(s) & Nakashima, Norihiro \\
\hline Citation & $\begin{array}{l}\text { Communications in A Igebra, 41(6), 2114-2131 } \\
\text { https://doi.org/10.1080/00927872.2012.654415 }\end{array}$ \\
\hline Issue Date & $2013-05-21$ \\
\hline Doc URL & http:/hdl.handle.net/2115/55722 \\
\hline Rights & $\begin{array}{l}\text { This is an Accepted Manuscript of an article published by Communications in A lgebra, 41(6) May 2013, } 2114.2131 . \\
\text { Communications in A Igebra is available online at: } \\
\text { http://www.tandfonline.com/doi full/10.1080/00927872.2012.654415\#.UjEZOD-ub3g }\end{array}$ \\
\hline Type & article (author version) \\
\hline File Information & nakashima Noetherian-Properties.pdf \\
\hline
\end{tabular}

Instructions for use 


\title{
THE NOETHERIAN PROPERTIES OF THE RINGS OF DIFFERENTIAL OPERATORS ON CENTRAL 2-ARRANGEMENTS
}

\author{
NORIHIRO NAKASHIMA
}

\begin{abstract}
Whereas Holm proved that the ring of differential operators on a generic hyperplane arrangement is finitely generated as an algebra, the problem of its Noetherian properties is still open. In this article, after proving that the ring of differential operators on a central arrangement is right Noetherian if and only if it is left Noetherian, we prove that the ring of differential operators on a central 2-arrangement is Noetherian. In addition, we prove that its graded ring associated to the order filtration is not Noetherian when the number of the consistuent hyperplanes is greater than 1 .
\end{abstract}

Key Words: Ring of differential operators;Noetherian property; Hyperplane arrangement.

2010 Mathematics Subject Classification: Primary 13N10; Secondary $32 \mathrm{~S} 22$.

\section{INTRODUCTION}

Let $K$ be a field of characteristic zero. For a commutative $K$-algebra $R$, we inductively define $K$-vector spaces of linear differential operators by

$$
\begin{aligned}
\mathscr{D}^{0}(R) & :=\left\{\theta \in \operatorname{End}_{K}(R) \mid a \in R, \theta a-a \theta=0\right\}, \\
\mathscr{D}^{m}(R) & :=\left\{\theta \in \operatorname{End}_{K}(R) \mid a \in R, \theta a-a \theta \in \mathscr{D}^{m-1}(R)\right\} \quad(m \geq 1) .
\end{aligned}
$$

We set $\mathscr{D}(R):=\bigcup_{m \geq 0} \mathscr{D}^{m}(R)$, and we call $\mathscr{D}(R)$ the ring of differential operators of $R$. Let $S:=K\left[x_{1}, \ldots, x_{n}\right]$ denote the polynomial ring. It is well known that the ring $\mathscr{D}(S)$ of differential operators of $S$ is the $n$-th Weyl algebra $K\left[x_{1}, \ldots, x_{n}\right]\left\langle\partial_{1}, \ldots, \partial_{n}\right\rangle$ where $\partial_{i}:=\frac{\partial}{\partial x_{i}}$ (see for example [5, Example 15.1.15] and [5, Corollaty 15.5.6]). We use the multi-index notations, for example, $\partial^{\alpha}:=\partial_{1}^{\alpha_{1}} \cdots \partial_{n}^{\alpha_{n}}$ and $|\boldsymbol{\alpha}|:=\alpha_{1}+\cdots+\alpha_{n}$ for $\boldsymbol{\alpha}=\left(\alpha_{1}, \ldots, \alpha_{n}\right) \in \mathbb{N}^{n}$. We set $\mathscr{D}^{(m)}(S):=\bigoplus_{|\boldsymbol{\alpha}|=m} S \partial^{\boldsymbol{\alpha}}$ for $m \geq 0$. We regard $\mathscr{D}^{(m)}(S)$ as a left $S$-module by the left product in the Weyl algebra. Then the Weyl algebra $\mathscr{D}(S)$ is decomposed into the direct sum of left $S$-modules $\mathscr{D}^{(m)}(S)$ of homogeneous differential operators: $\mathscr{D}(S)=\bigoplus_{m>0} \mathscr{D}^{(m)}(S)$.

There has been a lot of research on finiteness properties of the rings of differential operators. It is well known that $\mathscr{D}(R)$ is Noetherian, if $R$ is a regular domain (see [5, Theorem 15.1.20] and [5, Corollary 15.5.6]). There are some other important 
classes of algebras such that $\mathscr{D}(R)$ are Noetherian. For example, if $R$ is an integral domain of Krull dimension one, then $\mathscr{D}(R)$ is Noetherian (Muhasky [6] and SmithStafford [11]). Saito-Takahashi [10] showed that $\mathscr{D}(R)$ is right Noetherian if $R$ is an affine semigroup algebra. However, $\mathscr{D}(R)$ is not Noetherian in general. BernsteinGel'fand-Gel'fand [1] gave an example of a ring of differential operators that is neither Noetherian nor finitely generated.

Let $\mathcal{A}=\left\{H_{i} \mid i=1, \ldots, r\right\}$ be a central (hyperplane) arrangement (i.e., every hyperplane in $\mathcal{A}$ contains the origin) in $K^{n}$. Let $I$ be the defining ideal of $\mathcal{A}$. We consider the left $S$-module $\mathscr{D}^{(m)}(I)$ of differential operators homogeneous of order $m$ that preserve the ideal $I$. We call $\mathscr{D}^{(m)}(I)$ the modules of $\mathcal{A}$-differential operators. We find many results about the module $\mathscr{D}^{(1)}(I)$ of $\mathcal{A}$-derivations in a rich literature (see for example [8]). In contrast, there are only a few literatures about the modules of $\mathcal{A}$-differential operators of a higher order. Holm [4] proved that the ring of differential operators of the coordinate ring $S / I$ is finitely generated when $I$ is the ideal defining a generic hyperplane arrangement. In this paper, we will prove that $\mathscr{D}(S / I)$ is Noetherian if $n=2$.

In Section 3, we prove that $\mathscr{D}(S / I)$ is right Noetherian if and only if it is left Noetherian. Thus the Noetherian property of $\mathscr{D}(S / I)$ can be proved by the right or left Noetherian property.

In Section 4, we prove that $\mathscr{D}(S / I)$ is right Noetherian in the case $n=2$. This is the main result of this article. Let $R$ be a filtered ring, and $\mathcal{F}$ the filtration. If the graded ring associated to the filtration $\mathcal{F}$ of $R$ is right (left) Noetherian, then $R$ is right (left) Noetherian. However, the graded ring associated to the order filtration of $\mathscr{D}(S / I)$ is not Noetherian if $r \geq 2$ (Example 4.17). Hence we cannot take this convenient approach to prove the Noetherian property of $\mathscr{D}(S / I)$. The keys of the proof of the main result are Corollary 4.11 and Lemma 4.14.

There is a well-known basis for the module $\mathscr{D}^{(1)}(I)$ of $\mathcal{A}$-derivation (see for example $[8])$. Holm [3] studied the module $\mathscr{D}^{(m)}(I)$, and gave its basis for any order $m$. Let $\mathscr{D}(J)$ denote the subring of $\mathscr{D}(S)$ consisting of the operators preserving an ideal $J$. Holm [3],[4] showed that $\mathscr{D}(I)$ decomposes into the direct sum of $\mathscr{D}^{(m)}(I)$. For an ideal $J$, there is a ring isomorphism:

$$
\mathscr{D}(S / J) \simeq \mathscr{D}(J) / J \mathscr{D}(S)
$$

(see [5, Theorem 15.5.13]). Using these facts, we can write any element of $\mathscr{D}(S / I)$ as a linear combination of bases of the modules of $\mathcal{A}$-differential operators. This expression is useful to prove Corollary 4.11.

We consider a sequence of two-sided ideals of $\mathscr{D}(I)$ :

$$
I \mathscr{D}(S)=L_{r} \subseteq L_{r-1} \subseteq \cdots \subseteq L_{1} \subseteq L_{0}=\mathscr{D}(I) .
$$

We prove that $\mathscr{D}(I) / I \mathscr{D}(S)$ is right Noetherian by proving that each $L_{i-1} / L_{i}$ is right Noetherian $\mathscr{D}(I)$-module. To show the right Noetherian property of $L_{i-1} / L_{i}$, 
we study a module of lower order operators in $L_{i-1} / L_{i}$ and that of higher order operators separately.

We prove that the right $\mathscr{D}(I)$-module generated by the higher order operators in $L_{i-1} / L_{i}$ is Noetherian in Corollary 4.11, and that the module of lower order operators in $L_{i-1} / L_{i}$ is right Noetherian as a right $S$-module in Lemma 4.14. In this way, we see that $\mathscr{D}(S / I)$ is Noetherian.

\section{Differential operators on a CENTRAL ARRANGEMENT}

In this section, we fix some notation, and we refer to some facts used in Section 4. Let $\mathcal{A}=\left\{H_{i} \mid i=1, \ldots, r\right\}$ be a central arrangement in $K^{n}$. Fix a polynomial $p_{i}$ defining $H_{i}$, and put $Q:=p_{1} \cdots p_{r}$. Thus $Q$ is a product of certain homogeneous polynomials of degree 1 . We call $Q$ a defining polynomial of $\mathcal{A}$. Let $I$ denote the principal ideal of $S$ generated by $Q$.

For any ideal $J$ of $S$, we define an $S$-submodule $\mathscr{D}^{(m)}(J)$ of $\mathscr{D}^{(m)}(S)$ and a subring $\mathscr{D}(J)$ of $\mathscr{D}(S)$ by

$$
\begin{aligned}
\mathscr{D}^{(m)}(J) & :=\left\{\theta \in \mathscr{D}^{(m)}(S) \mid \theta(J) \subseteq J\right\}, \\
\mathscr{D}(J) & :=\{\theta \in \mathscr{D}(S) \mid \theta(J) \subseteq J\} .
\end{aligned}
$$

Among others, Holm [4] proved the following two propositions.

Proposition 2.1 (Proposition 4.3 in [4]). We have a direct sum

$$
\mathscr{D}(I)=\bigoplus_{m \geq 0} \mathscr{D}^{(m)}(I)
$$

as a left $S$-module.

Proposition 2.2 (Proposition 2.4 in [4]). Suppose that $f_{1}, \ldots, f_{k} \in S$ are coprime to one another. Then

$$
\mathscr{D}\left(\left\langle f_{1} \cdots f_{k}\right\rangle\right)=\bigcap_{i=1}^{k} \mathscr{D}\left(\left\langle f_{i}\right\rangle\right) .
$$

The following is well known (e.g., see [4, Proposition 2.3]).

Proposition 2.3. Let $J$ be the ideal of $S$ generated by $f_{1}, \ldots, f_{k}$, and let $\theta \in \mathscr{D}(S)$ be an operator of order $m \geq 1$. Then $\theta \in \mathscr{D}(J)$ if and only if $\theta\left(x^{\alpha} f_{j}\right) \in J$ for $|\boldsymbol{\alpha}| \leq m-1$ and $j=1, \ldots, k$.

We use the following lemma in Section 4. 
Lemma 2.4. Let $\delta \in \sum_{i=1}^{n} K \partial_{i}$, and let $f_{1}, \ldots, f_{k}$ be polynomials of degree 1 . If $k \leq m$, then

$$
\begin{aligned}
\delta^{m} f_{1} \ldots f_{k} & =\sum_{i=0}^{k}[m]_{i}\left(\sum_{\substack{\Lambda \subseteq\{1, \ldots, k\} \\
\sharp \Lambda=i}} \prod_{j \in \Lambda} \delta\left(f_{j}\right) \prod_{j \notin \Lambda} f_{j}\right) \delta^{m-i} \\
& =\sum_{i=0}^{k}[m]_{i}\left(\frac{1}{i !(k-i) !} \sum_{\sigma \in S_{k}} \delta\left(f_{\sigma(1)}\right) \ldots \delta\left(f_{\sigma(i)}\right) f_{\sigma(i+1)} \cdots f_{\sigma(k)}\right) \delta^{m-i},
\end{aligned}
$$

where $[m]_{0}:=1$ and $[m]_{i}:=m(m-1) \cdots(m-i+1)$ for $i \geq 1$.

Proof. For any $f \in S$, we see $\delta^{\ell} f=f \delta^{\ell}+\ell \delta(f) \delta^{\ell-1}$. We can prove the assertion by induction on $k$.

For a monomial $x^{\boldsymbol{\alpha}} \partial^{\boldsymbol{\beta}}$ in $\mathscr{D}(S)$, we define its total degree by

$$
\operatorname{totdeg}\left(x^{\boldsymbol{\alpha}} \partial^{\boldsymbol{\beta}}\right)=|\boldsymbol{\alpha}|-|\boldsymbol{\beta}| \text {. }
$$

For $\theta \in \mathscr{D}(S)$, we define the total degree of $\theta$ as the largest total degree of monomials in $\theta$. We consider $\mathscr{D}(S)$ a graded ring by the total degree.

The operator

$$
\varepsilon_{m}:=\sum_{|\boldsymbol{\alpha}|=m} \frac{m !}{\boldsymbol{\alpha} !} x^{\boldsymbol{\alpha}} \partial^{\boldsymbol{\alpha}}
$$

is called the Euler operator of order $m$ where $\boldsymbol{\alpha} !=\left(\alpha_{1} !\right) \cdots\left(\alpha_{n} !\right)$ for $\boldsymbol{\alpha}=\left(\alpha_{1}, \ldots, \alpha_{n}\right)$. Then $\varepsilon_{1}$ is the Euler derivation, and $\varepsilon_{m}=\varepsilon_{1}\left(\varepsilon_{1}-1\right) \cdots\left(\varepsilon_{1}-m+1\right)$ [4, Lemma 4.9].

\section{Right Noetherian property and Left Noetherian Property}

Let $Q=p_{1} \cdots p_{r}$ be a defining polynomial of a central arrangement $\mathcal{A}$, and let $I=Q S$. In this section, we will prove that the ring $\mathscr{D}(S / I)$ of differential operators is right Noetherian if and only if $\mathscr{D}(S / I)$ is left Noetherian. Recall that we have a ring isomorphism $\mathscr{D}(S / I) \simeq \mathscr{D}(I) / I \mathscr{D}(S)$ (see [5, Proposition 15.5.9 (ii)] and [5, Theorem 15.5.13]).

Let $0 \neq h \in S$, and set $J:=h S$. We denote by $K\left(x_{1}, \ldots, x_{n}\right)$ the field of fractions of $S$. Then $\mathscr{D}(S) \cap h \mathscr{D}(S) h^{-1} \subseteq K\left(x_{1}, \ldots, x_{n}\right)\left\langle\partial_{1}, \ldots, \partial_{n}\right\rangle$.

Lemma 3.1. As a ring,

$$
\mathscr{D}(J)=\mathscr{D}(S) \cap h \mathscr{D}(S) h^{-1} .
$$

Proof. Assume that $h \theta h^{-1} \in \mathscr{D}(S)$ with $\theta \in \mathscr{D}(S)$. For any $f \in S$,

$$
h \theta h^{-1}(h f)=h \theta(f) \in h S,
$$

which means $h \theta h^{-1} \in \mathscr{D}(J)$. 
Next we will prove the converse inclusion. Let $\theta \in \mathscr{D}(J)$. Since $h^{-1} \theta h \in$ $K\left(x_{1}, \ldots, x_{n}\right)\left\langle\partial_{1}, \ldots, \partial_{n}\right\rangle$, we can write

$$
h^{-1} \theta h=\sum_{\alpha} f_{\alpha} \partial^{\alpha}
$$

with $f_{\boldsymbol{\alpha}} \in K\left(x_{1}, \ldots, x_{n}\right)$. We show that $f_{\boldsymbol{\alpha}} \in S$ for all $\boldsymbol{\alpha}$ by induction on $|\boldsymbol{\alpha}|$.

Since

$$
f_{0}=h^{-1} \theta h(1)=h^{-1} \theta(h) \in h^{-1} h S=S,
$$

we have $f_{0} \in S$.

Assume that $f_{\boldsymbol{\alpha}} \in S$ for all $\boldsymbol{\alpha}$ with $|\boldsymbol{\alpha}|<m$. For $|\boldsymbol{\beta}|=m$,

$$
h^{-1} \theta h\left(x^{\boldsymbol{\beta}}\right)=\boldsymbol{\beta} ! f_{\boldsymbol{\beta}}+\sum_{|\boldsymbol{\alpha}|<m} f_{\boldsymbol{\alpha}} \partial^{\boldsymbol{\alpha}}\left(x^{\boldsymbol{\beta}}\right) .
$$

Since $\theta \in \mathscr{D}(J)$, we obtain

$$
h^{-1} \theta h\left(x^{\boldsymbol{\beta}}\right)=h^{-1} \theta\left(h x^{\boldsymbol{\beta}}\right) \in h^{-1} h S=S .
$$

Then $f_{\boldsymbol{\beta}} \in S$ by the induction hypothesis. Therefore we conclude that $h^{-1} \theta h=$ $\sum_{\alpha} f_{\alpha} \partial^{\alpha} \in \mathscr{D}(S)$.

Define an anti-automorphism ${ }^{t}: \mathscr{D}(S) \longrightarrow \mathscr{D}(S)$ by ${ }^{t} x_{i}=x_{i}{ }^{t} \partial_{i}=-\partial_{i}$ for $i=1, \ldots, n$ (we say that ${ }^{t}$ is an anti-automorphism if ${ }^{t}$ is an automorphism as a linear map, and if ${ }^{t}(\theta \eta)={ }^{t} \eta^{t} \theta$ for any $\left.\theta, \eta \in \mathscr{D}(S)\right)$. It is clear that ${ }^{t}\left({ }^{t} \theta\right)=\theta$ for any $\theta \in \mathscr{D}(S)$.

For $\theta \in \mathscr{D}(J)$, put $\theta^{*}:=h^{t} \theta h^{-1}$. Then

$$
\begin{aligned}
(\mathscr{D}(J))^{*} & =\left(\mathscr{D}(S) \cap h \mathscr{D}(S) h^{-1}\right)^{*} \\
& =h^{t}\left(\mathscr{D}(S) \cap h \mathscr{D}(S) h^{-1}\right) h^{-1} \\
& =h^{t} \mathscr{D}(S) h^{-1} \cap{ }^{t} \mathscr{D}(S) \\
& =h \mathscr{D}(S) h^{-1} \cap \mathscr{D}(S) \\
& =\mathscr{D}(J)
\end{aligned}
$$

by Lemma 3.1. Thus

$$
\text { * : } \mathscr{D}(J) \longrightarrow \mathscr{D}(J)
$$

is an anti-automorphism. If $h \theta \in J \mathscr{D}(S)$, then

$$
(h \theta)^{*}=h^{t}(h \theta) h^{-1}=h^{t} \theta h h^{-1}=h^{t} \theta \in J \mathscr{D}(S) .
$$

It is clear that $\theta=\left(\theta^{*}\right)^{*}$ for any $\theta \in \mathscr{D}(J)$. Hence we have $(J \mathscr{D}(S))^{*}=J \mathscr{D}(S)$. Therefore the anti-automorphism * induces an anti-automorphism

$$
\text { * }: \mathscr{D}(J) / J \mathscr{D}(S) \longrightarrow \mathscr{D}(J) / J \mathscr{D}(S) \text {. }
$$

The following is clear from the existence of the anti-automorphism * 
Theorem 3.2. The ring $\mathscr{D}(J) / J \mathscr{D}(S)$ is right Noetherian if and only if $\mathscr{D}(J) / J \mathscr{D}(S)$ is left Noetherian.

Corollary 3.3. Let I be the defining ideal of a central arrangement. Then the ring $\mathscr{D}(I) / I \mathscr{D}(S)$ is right Noetherian if and only if $\mathscr{D}(I) / I \mathscr{D}(S)$ is left Noetherian.

Remark 3.4. By the anti-isomorphism (3.1), it is also true that the ring $\mathscr{D}(J)$ (also $\mathscr{D}(I))$ is right Noetherian if and only if is left Noetherian.

It is known that some finiteness properties of rings of differential operators on irreducible affine algebraic varieties over an algebraically closed field (see [11, Theorem 2.5], [11, Proposition 7.3] and [11, Theorem 7.5]), whereas varieties of central hyperplane arrangements are reducible. Thus we cannot apply the results in [11].

\section{THE CASE $n=2$}

In this section, let $n=2$ and $S=K[x, y]$. We will prove that the $\operatorname{ring} \mathscr{D}(S / I) \simeq$ $\mathscr{D}(I) / I \mathscr{D}(S)$ of differential operators is Noetherian. We will also prove that, in contrast, the graded ring $\operatorname{Gr} \mathscr{D}(S / I)$ associated to the order filtration is not Noetherian when $r \geq 2$.

Put $P_{i}:=\frac{Q}{p_{i}}$ for $i=1, \ldots, r$, and define

$$
\delta_{i}:= \begin{cases}\partial_{y} & \text { if } p_{i}=a x \quad\left(a \in K^{\times}\right) \\ \partial_{x}+a_{i} \partial_{y} & \text { if } p_{i}=a\left(y-a_{i} x\right) \quad\left(a \in K^{\times}\right) .\end{cases}
$$

Then $\delta_{i}\left(p_{j}\right)=0$ if and only if $i=j$.

Proposition 4.1 (Paper III, Proposition 6.7 in [3], Proposition 4.14 in [12]). For any $m \geq 1, \mathscr{D}^{(m)}(I)$ is a free left $S$-module with basis

$$
\begin{aligned}
& \left\{\varepsilon_{m}, P_{1} \delta_{1}^{m}, \ldots, P_{m} \delta_{m}^{m}\right\} \text { if } m<r-1, \\
& \left\{P_{1} \delta_{1}^{m}, \ldots, P_{r} \delta_{r}^{m}\right\} \text { if } m=r-1, \\
& \left\{P_{1} \delta_{1}^{m}, \ldots, P_{r} \delta_{r}^{m}, Q \eta_{r+1}^{(m)}, \ldots, Q \eta_{m+1}^{(m)}\right\} \text { if } m>r-1,
\end{aligned}
$$

where the set $\left\{\delta_{1}^{m}, \ldots, \delta_{r}^{m}, \eta_{r+1}^{(m)}, \ldots, \eta_{m+1}^{(m)}\right\}$ forms a $K$-basis for $\sum_{|\boldsymbol{\alpha}|=m} K \partial^{\boldsymbol{\alpha}}$ if $m>$ $r-1$.

By Proposition 2.1, we have

$$
\begin{aligned}
\mathscr{D}(I)=S & \oplus\left(\bigoplus_{m=1}^{r-2}\left(S \varepsilon_{m} \oplus S P_{1} \delta_{1}^{m} \oplus \cdots \oplus S P_{m} \delta_{m}^{m}\right)\right) \\
& \oplus\left(\bigoplus_{m \geq r-1}\left(S P_{1} \delta_{1}^{m} \oplus \cdots \oplus S P_{r} \delta_{r}^{m} \oplus S Q \eta_{r+1}^{(m)} \oplus \cdots \oplus S Q \eta_{m+1}^{(m)}\right)\right) .
\end{aligned}
$$

For $i=1, \ldots, r$, define an additive group

$$
L_{i}:=\mathscr{D}(I) \cap\left(p_{1} \cdots p_{i}\right) \mathscr{D}(S) .
$$


Proposition 4.2. For $i=1, \ldots, r$, the additive group $L_{i}$ is a two-sided ideal of $\mathscr{D}(I)$.

Proof. It is clear that $L_{i}$ is a right ideal of $\mathscr{D}(I)$.

To prove that $L_{i}$ is a left ideal of $\mathscr{D}(I)$, by Proposition 2.1, we only need to prove that $\mathscr{D}^{(m)}(I) L_{i} \subseteq L_{i}$ for $m \geq 0$. Fix $\theta_{m} \in \mathscr{D}^{(m)}(I)$. For any $j=1, \ldots, i$, there exist $\eta_{\ell} \in D^{(\ell)}(S)$ such that

$$
\theta_{m} p_{j}=\eta_{0}+\cdots+\eta_{m}
$$

We prove that $\eta_{\ell} \in p_{j} \bigcap_{i^{\prime} \neq j} \mathscr{D}^{(\ell)}\left(p_{i^{\prime}} S\right) \subseteq \mathscr{D}^{(\ell)}(I)$ for $0 \leq \ell \leq m$ by induction on $\ell$.

In the case $\ell=0$, let (4.1) act on 1 . Then

$$
p_{j} S \ni \theta_{m}\left(p_{j}\right)=\eta_{0}
$$

because $\theta_{m} \in \mathscr{D}^{(\ell)}\left(p_{j} S\right)$ by Proposition 2.2. If $\ell \geq 1$, then it follows from the induction hypothesis that $\eta_{\ell}\left(x^{\boldsymbol{\alpha}}\right) \in p_{j} S$ for any $\boldsymbol{\alpha}$ with $|\boldsymbol{\alpha}|=\ell$ since

$$
p_{j} S \ni \theta_{m}\left(p_{j} x^{\boldsymbol{\alpha}}\right)=\eta_{0}\left(x^{\boldsymbol{\alpha}}\right)+\cdots+\eta_{\ell-1}\left(x^{\boldsymbol{\alpha}}\right)+\eta_{\ell}\left(x^{\boldsymbol{\alpha}}\right) .
$$

Therefore $\eta_{\ell} \in p_{j} \mathscr{D}^{(\ell)}(S)$. Write $\eta_{\ell}=p_{j} \eta_{\ell}^{\prime}$. For any $i^{\prime} \neq j$ and $|\boldsymbol{\alpha}|=\ell-1$, it also follows from the induction hypothesis that $p_{j} \eta_{\ell}^{\prime}\left(p_{i^{\prime}} x^{\alpha}\right)=\eta_{\ell}\left(p_{i^{\prime}} x^{\alpha}\right) \in p_{i^{\prime}} S$ since

$$
p_{i^{\prime}} S \ni \theta_{m}\left(p_{j} p_{i^{\prime}} x^{\alpha}\right)=\eta_{0}\left(p_{i^{\prime}} x^{\alpha}\right)+\cdots+\eta_{\ell-1}\left(p_{i^{\prime}} x^{\alpha}\right)+\eta_{\ell}\left(p_{i^{\prime}} x^{\alpha}\right) .
$$

Since $p_{j}$ and $p_{i^{\prime}}$ are coprime, we see that $\eta_{\ell}^{\prime}\left(p_{i^{\prime}} x^{\boldsymbol{\alpha}}\right) \in p_{i^{\prime}} S$. So $\eta_{\ell}^{\prime} \in \mathscr{D}\left(p_{i^{\prime}} S\right)$ by Proposition 2.3, and $\eta_{\ell} \in p_{j} \bigcap_{i^{\prime} \neq j} \mathscr{D}^{(\ell)}\left(p_{i^{\prime}} S\right)$. Thus $\theta_{m} p_{j} \in p_{j} \bigcap_{i^{\prime} \neq j} \mathscr{D}\left(p_{i^{\prime}} S\right)$. Then we conclude that

$$
\mathscr{D}(I) p_{1} \cdots p_{i} \mathscr{D}(S) \subseteq p_{1} \cdots p_{i} \mathscr{D}(S)
$$

By Proposition 2.1, $L_{i}$ is decomposed as follows:

$$
L_{i}=\bigoplus_{m \geq 0} L_{i}^{(m)}
$$

where $L_{i}^{(m)}:=\mathscr{D}^{(m)}(I) \cap\left(p_{1} \cdots p_{i}\right) \mathscr{D}^{(m)}(S)$. We consider a sequence

$$
I \mathscr{D}(S)=L_{r} \subseteq L_{r-1} \subseteq \cdots \subseteq L_{1} \subseteq L_{0}=\mathscr{D}(I)
$$

of two-sided ideals of $\mathscr{D}(I)$. If a right $\mathscr{D}(I)$-module $L_{i-1} / L_{i}$ is Noetherian for any $i$, then $\mathscr{D}(I) / I \mathscr{D}(S)$ is a right Noetherian ring. Now we fix $i$, and we will prove that $L_{i-1} / L_{i}$ is right Noetherian.

As a left $S$-module,

$$
L_{i-1} / L_{i}=\bigoplus_{m \geq 0}\left(L_{i-1}^{(m)}+L_{i} / L_{i}\right) \simeq \bigoplus_{m \geq 0}\left(L_{i-1}^{(m)} / L_{i}^{(m)}\right) .
$$


Put

$$
\begin{aligned}
& \left(L_{i-1} / L_{i}\right)^{<r-1}:=\bigoplus_{m<r-1}\left(L_{i-1}^{(m)} / L_{i}^{(m)}\right), \\
& \left(L_{i-1} / L_{i}\right)^{\geq r-1}:=\bigoplus_{m \geq r-1}\left(L_{i-1}^{(m)} / L_{i}^{(m)}\right) .
\end{aligned}
$$

Then $L_{i-1} / L_{i}$ is decomposed as a left $S$-module:

$$
L_{i-1} / L_{i}=\left(L_{i-1} / L_{i}\right)^{<r-1} \oplus\left(L_{i-1} / L_{i}\right)^{\geq r-1} .
$$

We will study $\left(L_{i-1} / L_{i}\right)^{<r-1}$ and $\left(L_{i-1} / L_{i}\right)^{\geq r-1}$ separately.

First we argue the part of order $\geq r-1$.

Lemma 4.3. Assume that $m \geq r-1$. As a left $S$-module,

$$
\begin{aligned}
L_{i}^{(m)}=S Q \delta_{1}^{m} \oplus \cdots \oplus S Q \delta_{i}^{m} \oplus S P_{i+1} \delta_{i+1}^{m} \oplus \cdots \oplus S P_{r} \delta_{r}^{m} \\
\oplus S Q \eta_{r+1}^{(m)} \oplus \cdots \oplus S Q \eta_{m+1}^{(m)} .
\end{aligned}
$$

Proof. Recall that $P_{i}=\frac{Q}{p_{i}}$. We see the assertion by Proposition 4.1 and the definition of $L_{i}$.

Proposition 4.4. For $m \geq 0$, we have

$$
L_{i}^{(m)} \cap S P_{i} \delta_{i}^{m}=S Q \delta_{i}^{m} \subseteq L_{i-1}^{(m)}
$$

as a left $S$-module. Hence

$$
\left(L_{i-1} / L_{i}\right)^{\geq r-1}=\bigoplus_{m \geq r-1}\left(S P_{i} \delta_{i}^{m}+L_{i}^{(m)} / L_{i}^{(m)}\right) \simeq \bigoplus_{m \geq r-1}\left(S P_{i} \delta_{i}^{m} / S Q \delta_{i}^{m}\right)
$$

as a left $S$-module.

Proof. By Lemma 4.3, $L_{i-1}^{(m)}=S P_{i} \delta_{i}^{m}+L_{i}^{(m)}$ for $m \geq r-1$. Then as a left $S$-module

$$
\left(L_{i-1} / L_{i}\right)^{\geq r-1}=\bigoplus_{m \geq r-1}\left(S P_{i} \delta_{i}^{m}+L_{i}^{(m)} / L_{i}^{(m)}\right) \simeq \bigoplus_{m \geq r-1}\left(S P_{i} \delta_{i}^{m} / L_{i}^{(m)} \cap S P_{i} \delta_{i}^{m}\right) .
$$

It remains to prove that

$$
L_{i}^{(m)} \cap S P_{i} \delta_{i}^{m}=S Q \delta_{i}^{m} \subseteq L_{i-1}^{(m)}
$$

for $m \geq 0$. It is clear that $S Q \delta_{i}^{m} \subseteq L_{i}^{(m)} \cap S P_{i} \delta_{i}^{m}$. Conversely, suppose that $f P_{i} \delta_{i}^{m} \in$ $L_{i}^{(m)}$ with $f \in S$. Then $f P_{i} \delta_{i}^{m} \in p_{1} \cdots p_{i} \mathscr{D}^{(m)}(S)$. Since the polynomials $p_{i}, \ldots, p_{r}$ are coprime to one another, we have $f \in p_{i} S$. Thus $L_{i}^{(m)} \cap S P_{i} \delta_{i}^{m} \subseteq S Q \delta_{i}^{m}$.

We define a left $S$-module

$$
E_{i}:=\bigoplus_{m \geq 0}\left(S P_{i} \delta_{i}^{m}+L_{i}^{(m)} / L_{i}^{(m)}\right) \simeq \bigoplus_{m \geq 0}\left(S P_{i} \delta_{i}^{m} / S Q \delta_{i}^{m}\right) .
$$


Note that $\left(S P_{i}+L_{i}^{(0)}\right) / L_{i}^{(0)} \simeq S P_{i} / S P_{i} \cap L_{i}^{(0)}=S P_{i} / S Q$. By Proposition 4.4, we may identify $\left(L_{i-1} / L_{i}\right)^{\geq r-1}$ with the $S$-submodule of $E_{i}$ of order $m \geq r-1$. It is clear that $\delta_{i} p_{i}=p_{i} \delta_{i}$ for $i=1, \ldots, r$. For $g \in S$, we have

$$
P_{i} \delta_{i}^{m}(Q g)=Q \delta_{i}^{m}\left(\frac{Q}{p_{i}} g\right) \in Q S .
$$

Proposition 4.1 says already that $P_{i} \delta_{i}^{m} \in \mathscr{D}^{(m)}(I)$. Since there is so much notation, we should remind us that $P_{i} \delta_{i}^{m} \in\left(p_{1} \cdots p_{i-1}\right) \mathscr{D}(S)$, and so $P_{i} \delta_{i}^{m} \in L_{i-1}$. Hence $E_{i}$ is a left $S$-submodule of $L_{i-1} / L_{i}$. Moreover, the following proposition is true:

Proposition 4.5. The module $E_{i}$ is a right $\mathscr{D}(I)$-submodule of $L_{i-1} / L_{i}$.

Proof. We only need to check the right multiplication by the elements of $S$ and the bases for $\mathscr{D}(I)$ in Proposition 4.1.

Let $m \geq 1$. For $g \in S$, we have

$$
\delta_{i}^{m} \cdot g \in S+\sum_{\ell=1}^{m} S \delta_{i}^{\ell},
$$

and hence $P_{i} \delta_{i}^{m} \cdot S \subseteq E_{i}$.

We show that $E_{i}$ is closed under the right action of the elements of bases for $\mathscr{D}(I)$. We only need to check the right multiplication by the elements $P_{i} \delta_{i}^{\ell}, P_{j} \delta_{j}^{m}(j \neq$ $i), \varepsilon_{\ell}, Q \eta_{j}^{(\ell)}$. For $m \geq 1$, we have

$$
\begin{gathered}
P_{i} \delta_{i}^{m} \cdot P_{i} \delta_{i}^{\ell}=P_{i}\left(\delta_{i}^{m} \cdot P_{i}\right) \delta_{i}^{\ell} \in \bigoplus_{m \geq 0}\left(S P_{i} \delta_{i}^{m}+L_{i}^{(m)}\right), \\
P_{i} \delta_{i}^{m} \cdot P_{j} \delta_{j}^{\ell}=Q \delta_{i}^{m} \cdot \frac{P_{j}}{p_{i}} \delta_{j}^{l} \in \mathscr{D}(I) \cap\left(p_{1} \cdots p_{i}\right) \mathscr{D}(S)=L_{i}, \\
P_{i} \delta_{i}^{m} \cdot Q \eta_{j}^{(\ell)}=Q \delta_{i}^{m} \cdot \frac{Q}{p_{i}} \eta_{j}^{(\ell)} \in \mathscr{D}(I) \cap\left(p_{1} \cdots p_{i}\right) \mathscr{D}(S)=L_{i}
\end{gathered}
$$

from the inclusion $P_{i} \delta_{i}^{m} \cdot S \subseteq E_{i}$. It remains to show that $E_{i}$ is closed under the right multiplication by $\varepsilon_{\ell}=\varepsilon_{1}\left(\varepsilon_{1}-1\right) \cdots\left(\varepsilon_{1}-\ell+1\right)$. We consider the Euler derivation $\varepsilon_{1}$. We may assume $p_{i}=y-a x\left(a \in K^{\times}\right)$. Recall $\delta_{i}=a^{-1} \partial_{x}+\partial_{y}$. Since

$$
\begin{aligned}
\varepsilon_{1} & =x \partial_{x}+y \partial_{y} \\
& =a^{-1} a x \partial_{x}+y \partial_{y}+a^{-1} y \partial_{x}-a^{-1} y \partial_{x} \\
& =a^{-1}(a x-y) \partial_{x}+y\left(\partial_{y}+a^{-1} \partial_{x}\right) \\
& =-a^{-1} p_{i} \partial_{x}+y \delta_{i},
\end{aligned}
$$

we have, for any $m \geq 0$,

$$
P_{i} \delta_{i}^{m} \cdot \varepsilon_{1}=P_{i} \delta_{i}^{m} \cdot\left(-a^{-1} p_{i} \partial_{x}+y \delta_{i}\right)=-a^{-1} Q \delta_{i}^{m} \partial_{x}+y P_{i} \delta_{i}^{m+1}+m P_{i} \delta_{i}^{m} .
$$


We see that $-a^{-1} Q \delta_{i}^{m} \partial_{x} \in L_{i}$, and that the remaining terms belong to $S P_{i} \delta_{i}^{m+1}$ and $S P_{i} \delta_{i}^{m}$, respectively. It follows that

$$
P_{i} \delta_{i}^{m} \cdot \varepsilon_{\ell} \in \bigoplus_{m \geq 0}\left(S P_{i} \delta_{i}^{m}+L_{i}^{(m)}\right) .
$$

Hence $E_{i} \cdot \varepsilon_{\ell} \subseteq E_{i}$. This completes the assertion.

As a left $S$-module,

$$
\left(L_{i-1} / L_{i}\right)^{\geq r-1} \subseteq E_{i} .
$$

The right $\mathscr{D}(I)$-module generated by $\left(L_{i-1} / L_{i}\right)^{\geq r-1}$ is a $\mathscr{D}(I)$-submodule of $E_{i}$ by Proposition 4.5:

$$
\left(L_{i-1} / L_{i}\right)^{\geq r-1} \cdot \mathscr{D}(I) \subseteq E_{i} .
$$

If we prove that $E_{i}$ is a right Noetherian $\mathscr{D}(I)$-module, then $\left(L_{i-1} / L_{i}\right)^{\geq r-1} \cdot \mathscr{D}(I)$ is Noetherian as a $\mathscr{D}(I)$-module. We will prove that $E_{i}$ is a right Noetherian $\mathscr{D}(I)$ module.

We define a left action of $S / p_{i} S$ on $E_{i}$ by

$$
\bar{f} \cdot \bar{\theta}=\overline{f \theta}
$$

for $\bar{f} \in S / p_{i} S$ and $\bar{\theta} \in E_{i}$. This is well-defined, since

$$
f \theta-g \theta^{\prime}=\frac{(f-g)\left(\theta+\theta^{\prime}\right)}{2}+\frac{(f+g)\left(\theta-\theta^{\prime}\right)}{2} \in L_{i}
$$

for $f, g \in S$ and $\theta, \theta^{\prime} \in \bigoplus_{m \geq 0}\left(S P_{i} \delta_{i}^{m}+L_{i}^{(m)}\right)$ with $f-g \in p_{i} S$ and $\theta-\theta^{\prime} \in L_{i}$. Thus $E_{i}$ is a left $S / p_{i} S$-module. We may assume that $p_{i}=y-a x$ with $a \neq 0$. Then $E_{i}$ is a $K$-vector space with a basis $\left\{\bar{y}^{\alpha} \cdot \overline{P_{i} \delta_{i}^{m}} \mid \alpha \in \mathbb{N}, m \geq 0\right\}$.

Define an exponent by

$$
\exp \left(\bar{y}^{\alpha} \cdot \overline{P_{i} \delta_{i}^{m}}\right):=(\alpha+r-1, m)
$$

for an element of the basis above. We call $\bar{y}^{\alpha} \cdot \overline{P_{i} \delta_{i}^{m}}$ a monomial of $E_{i}$. Let $\overline{\theta_{1}}$ and $\overline{\theta_{2}}$ be two monomials of $E_{i}$ with $\exp \left(\overline{\theta_{1}}\right)=\left(\alpha_{1}, m_{1}\right)$ and $\exp \left(\overline{\theta_{2}}\right)=\left(\alpha_{2}, m_{2}\right)$. We define a total order in the set of exponents of monomials by

$$
\exp \left(\overline{\theta_{1}}\right)<\exp \left(\overline{\theta_{2}}\right)
$$

if $m_{1}<m_{2}$, or if $m_{1}=m_{2}$ and $\alpha_{1}<\alpha_{2}$. For $\bar{\theta} \in E_{i}$, write $\bar{\theta}$ as a linear combination of monomials. Then we define an exponent of $\bar{\theta}$ as the largest exponent of a monomial in $\bar{\theta}$ with a nonzero coefficient, and we denote it by $\exp (\bar{\theta})$. For a subset $X$ of $E_{i}$, set

$$
\operatorname{Exp}(X):=\{\exp (\bar{\theta}) \mid \bar{\theta} \in X\}
$$

Throughout the remaining of this section, we write $\theta \in E_{i}$ instead of $\bar{\theta}$ for simplicity. 
Lemma 4.6. Let $M_{1}, M_{2}$ be right $\mathscr{D}(I)$-submodules of $E_{i}$. If $M_{1} \subseteq M_{2}$ and $\operatorname{Exp}\left(M_{1}\right)=$ $\operatorname{Exp}\left(M_{2}\right)$, then

$$
M_{1}=M_{2} .
$$

Proof. Suppose that $M_{1} \subsetneq M_{2}$. We can take an element $\theta \in M_{2} \backslash M_{1}$ such that $\exp (\theta)$ is the smallest exponent in $M_{2} \backslash M_{1}$.

Since $\exp (\theta) \in \operatorname{Exp}\left(M_{2}\right)=\operatorname{Exp}\left(M_{1}\right)$, there exists $\eta \in M_{1}$ such that $\exp (\eta)=$ $\exp (\theta)$. Then

$$
\exp (\theta-c \eta)<\exp (\theta)
$$

for some $c \in K^{\times}$. We have $\theta-c \eta \in M_{2} \backslash M_{1}$ since $\theta \notin M_{1}$. This is a contradiction to the minimality.

Lemma 4.7. Let $M \neq 0$ be a right $\mathscr{D}(I)$-submodule of $E_{i}$. If $(k, m) \in \operatorname{Exp}(M)$, then

$$
\left\{(k+a, m),\left(k+b, m+m^{\prime}\right) \mid a \geq 0, b \geq r-1, m^{\prime} \geq 1\right\} \subset \operatorname{Exp}(M) .
$$

Proof. By the assumption, there exists $\theta \in M$ such that $\exp (\theta)=(k, m)$. Put $\alpha:=$ $k-r+1$, and write $\theta=y^{\alpha} P_{i} \delta_{i}^{m}+\theta^{\prime}$ with $\exp \left(\theta^{\prime}\right)<\exp \left(y^{\alpha} P_{i} \delta_{i}^{m}\right)$. The multiplication $\theta \cdot y^{a}$ belongs to $M$, since $S \subseteq \mathscr{D}(I)$. Thus we see that $(k+a, m) \in \operatorname{Exp}(M)$ for all $a \geq 0$.

Fix $1 \leq j \neq i \leq r, b \geq r-1$, and $m^{\prime} \geq 1$. We can write

$$
y^{\alpha} P_{i} \delta_{i}^{m} \cdot p_{j}^{b-r+1} P_{i} \delta_{i}^{m^{\prime}}=y^{\alpha}\left(p_{j}^{b-r+1} P_{i}\right) P_{i} \delta_{i}^{m+m^{\prime}}+\eta .
$$

for some $\eta \in E_{i}$ with $\exp (\eta)<\exp \left(y^{\alpha}\left(p_{j}^{b-r+1} P_{i}\right) P_{i} \delta_{i}^{m+m^{\prime}}\right)$. Since $p_{j}^{b-r+1} P_{i} \notin p_{i} S$, we see that $\exp \left(\theta \cdot p_{j}^{b-r+1} P_{i} \delta_{i}^{m^{\prime}}\right)=\left(k+b, m+m^{\prime}\right)$. Therefore $\left(k+b, m+m^{\prime}\right) \in \operatorname{Exp}(M)$ since $\theta \cdot p_{j}^{b-r+1} P_{i} \delta_{i}^{m^{\prime}} \in M$.

Now we induce the total degree $(2.1)$ of $\mathscr{D}(S)$ to those of $\mathscr{D}(I)$ and $E_{i}$. Then $E_{i}$ becomes a graded $\mathscr{D}(I)$-module by the total degree. For monomials of $E_{i}$, we denote the total degree by

$$
\operatorname{totdeg}\left(y^{\alpha}\right)=\alpha, \operatorname{totdeg}\left(y^{\alpha^{\prime}} \cdot P_{i} \delta_{i}^{m}\right)=\alpha^{\prime}+r-1-m .
$$

Let $M$ be a right graded $\mathscr{D}(I)$-submodule of $E_{i}$. Set $X_{j}:=\{\ell \mid(j, \ell) \in \operatorname{Exp}(M)\}$. From Lemma 4.7, there exists the smallest integer $j$ with $\sharp X_{j}=\infty$. Put $s:=s_{M}:=$ $\min \left\{j \mid \sharp X_{j}=\infty\right\}$, and set $M_{s}:=\{\theta \in M \mid \exp (\theta)=(s, \ell)$ for some $\ell\}$. Then it is clear that $s \geq r-1$.

Let $\theta_{m} \in \bar{M}$ be a homogeneous operator satisfying $\exp \left(\theta_{m}\right)=(s, m)$ with $m \geq s$. Since $m-s+r-1>0$, we can write

$$
\theta_{m}=\sum_{\ell=0}^{s-r+1} a_{\ell} y^{s-r+1-\ell} P_{i} \delta_{i}^{m-\ell} \quad\left(a_{\ell} \in K\right) .
$$


We may assume that $a_{0}=1$. Set $\Omega:=\{1, \ldots, i-1, i+1, \ldots, r\}$. For $0 \leq \ell \leq s-r+1$, we write

$$
\begin{aligned}
& \delta_{i}^{m-\ell} P_{i} \\
& =\sum_{\ell^{\prime}=0}^{r-1}[m-\ell]_{\ell^{\prime}}\left(\frac{1}{\ell^{\prime} !\left(r-1-\ell^{\prime}\right) !} \sum_{\sigma \in S^{\Omega}} \delta\left(p_{\sigma(1)}\right) \cdots \delta\left(p_{\sigma\left(\ell^{\prime}\right)}\right) p_{\sigma\left(\ell^{\prime}+1\right)} \cdots p_{\sigma(r)}\right) \delta_{i}^{m-\ell-\ell^{\prime}} \\
& \equiv \sum_{\ell^{\prime}=0}^{r-1}[m-\ell]_{\ell^{\prime}} d_{\ell^{\prime}} y^{r-1-\ell^{\prime}} \delta_{i}^{m-\ell-\ell^{\prime}} \quad\left(\bmod p_{i} D(S)\right),
\end{aligned}
$$

for some $d_{\ell^{\prime}} \in K$ by Lemma 2.4. It should be argued that $d_{0} \neq 0$ and $d_{r-1} \neq 0$. We can write

$$
\delta_{i}^{m-\ell} P_{i}=\sum_{\ell^{\prime}=0}^{r-1} f_{\ell^{\prime}} \delta_{i}^{m-\ell-\ell^{\prime}}
$$

in the Weyl algebra.

First we argue $d_{0} \neq 0$. The polynomial coefficient $f_{0}$ of $\delta_{i}^{m-\ell}$ is the polynomial $P_{i}$, and $P_{i} \not \equiv 0\left(\bmod p_{i}\right)$ since $p_{1}, \ldots, p_{r}$ are coprime to one another. By the definition $d_{0}$, we have $P_{i} \equiv d_{0} y^{r-1}\left(\bmod p_{i}\right)$. This implies $d_{0} \neq 0$. Next we argue $d_{r-1} \neq 0$, The coefficient $f_{r-1}$ of $\delta_{i}^{m-\ell-r+1}$ is equal to $\delta_{i}\left(p_{1}\right) \cdots \delta_{i}\left(p_{i}\right) \cdots \delta_{i}\left(p_{r}\right)$. Since $\delta_{i}\left(p_{j}\right)=0$ if and only if $i=j$, we have $\delta_{i}\left(p_{1}\right) \cdots \delta_{i}\left(p_{i}\right) \cdots \delta_{i}\left(p_{r}\right) \neq 0$. This means $d_{r-1} \neq 0$.

Therefore we obtain $d_{0} \neq 0$ and $d_{r-1} \neq 0$. Then

$$
\begin{aligned}
\theta_{m} \cdot P_{i} \delta_{i}^{m^{\prime}} & =\sum_{\ell=0}^{s-r+1} a_{\ell} y^{s-r+1-\ell} P_{i}\left(\delta_{i}^{m-\ell} P_{i}\right) \delta_{i}^{m^{\prime}} \\
& =\sum_{\ell=0}^{s-r+1} \sum_{\ell^{\prime}=0}^{r-1} a_{\ell}[m-\ell]_{\ell^{\prime}} d_{\ell^{\prime}} y^{k-\ell-\ell^{\prime}} P_{i} \delta_{i}^{m-\ell-\ell^{\prime}} \\
& =\sum_{t=0}^{s} c_{t} y^{s-t} P_{i} \delta_{i}^{m+m^{\prime}-t}
\end{aligned}
$$

where

$$
c_{t}:=\sum_{\substack{0 \leq \ell \leq s-r+1 \\ 0 \leq \ell^{\prime} \leq r-1 \\ \ell+\ell^{\prime}=t}} a_{\ell}[m-\ell]_{\ell^{\prime}} d_{\ell^{\prime}}
$$

for $0 \leq t \leq s$. We remark that $c_{t}$ does not depend on $m^{\prime}$. Put $m_{0}:=\max \{\ell \mid$ $(s-1, \ell) \in \operatorname{Exp}(M)\}+s$. 
Lemma 4.8. For $1 \leq j \leq r$, there exist operators $\theta_{m_{1}}, \ldots, \theta_{m_{j}} \in M_{s}$ such that

$$
\operatorname{rank}\left(\begin{array}{ccc}
c_{0}^{(1)} & \cdots & c_{0}^{(j)} \\
\vdots & & \vdots \\
c_{r-1}^{(1)} & \cdots & c_{r-1}^{(j)}
\end{array}\right)=j,
$$

and $m_{0}<m_{1}<\cdots<m_{j}$, where $c_{t}^{(j)}$ for $\theta_{m_{j}}$ has been defined in (4.7).

Proof. We prove the assertion by induction. It is clear in the case $j=1$.

Let $1<j<r$. Assume that there exist $\theta_{m_{1}}, \ldots, \theta_{m_{j}} \in M_{s}\left(m_{1}<\cdots<m_{j}\right)$ satisfying the condition (4.8).

For $m>m_{j}$, put a vector

$$
\boldsymbol{w}:=\left(y^{s} P_{i} \delta_{i}^{m+m^{\prime}}, y^{s-1} P_{i} \delta_{i}^{m+m^{\prime}-1}, \ldots, P_{i} \delta_{i}^{m+m^{\prime}-s}\right),
$$

and put an $(s+1) \times(s-r+j+2)$ matrix

$$
A:=\left(\begin{array}{cccccccc}
c_{0}^{(1)} & \cdots & c_{0}^{(j)} & d_{0} & 0 & 0 & \cdots & 0 \\
c_{1}^{(1)} & \cdots & c_{1}^{(j)} & {[m]_{1} d_{1}} & d_{0} & 0 & \cdots & 0 \\
c_{2}^{(1)} & \cdots & c_{2}^{(r-1)} & {[m]_{2} d_{2}} & {[m-1]_{1} d_{1}} & d_{0} & \ddots & 0 \\
\vdots & & \vdots & \vdots & & \ddots & \ddots & \vdots \\
c_{r-2}^{(1)} & \cdots & c_{r-2}^{(j)} & {[m]_{r-2} d_{r-2}} & & & & 0 \\
c_{r-1}^{(1)} & \cdots & c_{r-1}^{(j)} & {[m]_{r-1} d_{r-1}} & {[m-1]_{r-2} d_{r-2}} & & & d_{0} \\
\vdots & & \vdots & 0 & {[m-1]_{r-1} d_{r-1}} & & & \vdots \\
\vdots & & \vdots & 0 & 0 & \ddots & & \vdots \\
\vdots & & \vdots & \vdots & & \ddots & \ddots & \vdots \\
c_{s}^{(1)} & \cdots & c_{s}^{(j)} & 0 & \cdots & \cdots & 0 & {[m-s+r-1]_{r-1} d_{r-1}}
\end{array}\right) .
$$

We consider $m$ as a variable. By the induction hypothesis, there exists a nonzero $j$-minor of the matrix in (4.8). We denote by $B$ the matrix of this $j$-minor. We take the lowest $s-r$ rows of $A$ and $j$ rows from the remaining $r-1$ rows of $A$ so that we get the $(s-r+j+2)$-minor $C$ whose matrix contains the matrix $B$. The coefficient of the leading term of $C$ is the determinant of $B$. Thus $C$ is not zero as a polynomial in variable $m$, and hence the solutions of $C=0$ is finite. Because of this, the number of $m$ with $\operatorname{rank}(A)<s-r+j+2$ is finite. Hence we can take a positive integer $m>m_{j}$ such that $\exp \left(\theta_{m}\right) \in M_{s}$, and $\operatorname{rank}(A)=s-r+j+2$.

We write $\theta_{m}=\sum_{\ell=0}^{s-r+1} a_{\ell} y^{s-r+1-\ell} P_{i} \delta_{i}^{m-\ell}$ in the same way as in (4.5). Put

$$
\boldsymbol{v}:=\left(\lambda_{1}, \ldots, \lambda_{j}, \lambda_{j+1} a_{0}, \ldots, \lambda_{j+1} a_{s-r+1}\right) .
$$

Then

$$
\boldsymbol{w} A^{t} \boldsymbol{v}=\lambda_{1} \theta_{m_{1}} \cdot P_{i} \delta_{i}^{m_{1}^{\prime}}+\cdots+\lambda_{j} \theta_{m_{j}} \cdot P_{i} \delta_{i}^{m_{j}^{\prime}}+\lambda_{j+1} \theta_{m} \cdot P_{i} \delta_{i}^{m^{\prime}}
$$


with $m_{1}+m_{1}^{\prime}=\cdots=m_{j}+m_{j}^{\prime}=m+m^{\prime}$. If $\boldsymbol{w} A^{t} \boldsymbol{v}=0$, then $\boldsymbol{v}=0$ since $\operatorname{rank}(A)=s-r+j+2$. Therefore $\left\{\theta_{m_{1}} \cdot P_{i} \delta_{i}^{m_{1}^{\prime}}, \ldots, \theta_{m_{j}} \cdot P_{i} \delta_{i}^{m_{j}^{\prime}}, \theta_{m} \cdot P_{i} \delta_{i}^{m^{\prime}}\right\}$ is linearly independent over $K$.

Put $\theta_{m_{j+1}}:=\theta_{m}$, and suppose that

$$
\operatorname{rank}\left(\begin{array}{ccc}
c_{0}^{(1)} & \cdots & c_{0}^{(j+1)} \\
\vdots & & \vdots \\
c_{r-1}^{(1)} & \cdots & c_{r-1}^{(j+1)}
\end{array}\right)<j+1 .
$$

Then there exists $\left(\lambda_{1}, \ldots, \lambda_{j+1}\right) \in K^{j+1} \backslash\{\mathbf{0}\}$ such that

$$
\lambda_{1}{ }^{t}\left(c_{0}^{(1)}, \ldots, c_{r-1}^{(1)}\right)+\cdots+\lambda_{j+1}{ }^{t}\left(c_{0}^{(j+1)}, \ldots, c_{r-1}^{(j+1)}\right)=\mathbf{0} .
$$

Since $\left\{\theta_{m_{1}} \cdot P_{i} \delta_{i}^{m_{1}^{\prime}}, \ldots, \theta_{m_{j+1}} \cdot P_{i} \delta_{i}^{m_{j+1}^{\prime}}\right\}$ is linearly independent, we have

$$
\sum_{k=0}^{j+1} \lambda_{k} \theta_{m_{k}} \cdot P_{i} \delta_{i}^{m_{k}^{\prime}} \neq 0 .
$$

Hence we can write $\exp \left(\sum_{k=0}^{j+1} \lambda_{k} \theta_{m_{k}} \cdot P_{i} \delta_{i}^{m_{k}^{\prime}}\right)=(\alpha, \beta)$ for some $\alpha<s$ and $\beta>$ $m_{j+1}+m_{j+1}^{\prime}-s>m_{0}-s=\max \{\ell \mid(s-1, \ell) \in \operatorname{Exp}(M)\}$ by (4.9) and (4.10). This is a contradiction.

Let $M$ be a right graded $\mathscr{D}(I)$-submodule of $E_{i}$. For a nonnegative integer $\ell$ with $(k, \ell) \in \operatorname{Exp}(M)$ for some $k$, we define an integer $t_{\ell}$ by

$$
t_{\ell}:=\min \{k \mid(k, \ell) \in \operatorname{Exp}(M)\} .
$$

By Lemma 4.8, there exist operators $\theta_{m_{1}}, \ldots, \theta_{m_{r}} \in M_{s}$ satisfying the condition (4.8). We denote by $N$ the right submodule of $M$ generated by the operators $\theta_{m_{1}}, \ldots, \theta_{m_{r}}$.

Lemma 4.9. There exists a positive integer $n_{0}$ such that, for any $m \geq n_{0}$,

$$
(s, m) \in \operatorname{Exp}(N) \text { and } t_{m}=s .
$$

Proof. By Lemma 4.8, there exist $\theta_{m_{1}}, \ldots, \theta_{m_{r}} \in M_{s}$ such that

$$
\operatorname{rank}\left(\begin{array}{ccc}
c_{0}^{(1)} & \cdots & c_{0}^{(r)} \\
\vdots & & \vdots \\
c_{r-1}^{(1)} & \cdots & c_{r-1}^{(r)}
\end{array}\right)=r \text {, and } \operatorname{rank}\left(\begin{array}{ccc}
c_{0}^{(1)} & \cdots & c_{0}^{(r)} \\
\vdots & & \vdots \\
c_{r-2}^{(1)} & \cdots & c_{r-2}^{(r)}
\end{array}\right)<r .
$$

Then there exists a nonzero vector $\left(\lambda_{1}, \ldots, \lambda_{r}\right) \in K^{r} \backslash\{\mathbf{0}\}$ such that

$$
\lambda_{1}{ }^{t}\left(c_{0}^{(1)}, \ldots, c_{r-2}^{(1)}\right)+\cdots+\lambda_{r}{ }^{t}\left(c_{0}^{(r)}, \ldots, c_{r-2}^{(r)}\right)=\mathbf{0},
$$

and

$$
\lambda_{1} c_{r-1}^{(1)}+\cdots+\lambda_{r} c_{r-1}^{(r)} \neq 0
$$


Put $\theta:=\sum_{k=0}^{r} \lambda_{k} \theta_{m_{k}} \cdot P_{i} \delta_{i}^{m_{k}^{\prime}} \in N$ with $m_{1}+m_{1}^{\prime}=\cdots=m_{r}+m_{r}^{\prime}$. It follows that $\exp (\theta)=\left(s, m_{r}+m_{r}^{\prime}-r+1\right)$. Put $n_{0}=m_{r}-r+1$, and put $m_{r}^{\prime}=m-n_{0}$ for any $m \geq n_{0}$. Thus

$$
(s, m)=\left(s, m_{r}+m_{r}^{\prime}-r+1\right)=\exp (\theta) \in \operatorname{Exp}(N) .
$$

It remains to prove that $t_{m}=s$. We have $t_{m} \geq s$ since $m \geq n_{0} \geq m_{0}-s=$ $\max \{\ell \mid(s-1, \ell) \in \operatorname{Exp}(M)\}$. Conversely we have $t_{m} \leq s$ since $(s, m) \in \operatorname{Exp}(M)$, as required.

Let $R$ be a graded ring. A right graded $R$-module $M$ is said to be right grNoetherian, if $M$ satisfies the ascending chain condition for graded submodules of $M$. It is straightforward to verify that $M$ is right gr-Noetherian if and only if each graded submodule of $M$ is finitely generated.

Proposition 4.10. The right $\mathscr{D}(I)$-module $E_{i}$ is right Noetherian.

Proof. Recall that $\mathscr{D}(I)$ is a graded ring by the total degree, and that $E_{i}$ is a graded $\mathscr{D}(I)$-module. By [7, Theorem II.3.5], it is enough to prove that $E_{i}$ is right grNoetherian. Let $M$ be a right graded $\mathscr{D}(I)$-submodule of $E_{i}$. We will prove that $M$ is finitely generated.

Let $n_{0}$ be the integer satsfying Lemma 4.9. Set

$$
G:=\left\{\left(t_{\ell}, \ell\right) \mid \ell<n_{0} \text { and }(k, \ell) \in \operatorname{Exp}(M) \text { for some } k\right\} .
$$

Then $G$ is a finite set. Fix an operator $\theta_{\left(t_{\ell}, \ell\right)} \in M$ for $\left(t_{\ell}, \ell\right) \in G$, and set

$$
\bar{G}:=\left\{\theta_{\left(t_{\ell}, \ell\right)} \in M \mid\left(t_{\ell}, \ell\right) \in G\right\} .
$$

Then $\bar{G}$ is also a finite set. We denote by $M^{\prime}$ the right $\mathscr{D}(I)$-module generated by $\bar{G}$ and $N$. Then $M^{\prime}$ is finitely generated and $M^{\prime} \subseteq M$.

Let $(k, m) \in \operatorname{Exp}(M)$, then $k \geq t_{m}$. If $m<n_{0}$, then $\left(t_{m}, m\right) \in G \subseteq \operatorname{Exp}\left(M^{\prime}\right)$ by the definitions of $t_{m}$ and $G$. We have $(k, m)=\left(t_{m}+k-t_{m}, m\right) \in \operatorname{Exp}\left(M^{\prime}\right)$ by Lemma 4.7.

If $m \geq n_{0}$, then $(s, m) \in \operatorname{Exp}\left(M^{\prime}\right)$ by Lemma 4.9. It follows from Lemma 4.7 that $(k, m)=(s+k-s, m) \in \operatorname{Exp}\left(M^{\prime}\right)$. Hence $\operatorname{Exp}\left(M^{\prime}\right)=\operatorname{Exp}(M)$. The assertion follows from Lemma 4.6.

Corollary 4.11. The right $\mathscr{D}(I)$-module

$$
\left(L_{i-1} / L_{i}\right)^{\geq r-1} \cdot \mathscr{D}(I)=\left(L_{i-1}^{\geq r-1} \cdot \mathscr{D}(I)+L_{i} / L_{i}\right)
$$

is right Noetherian.

Next we study the $S$-module $\left(L_{i-1} / L_{i}\right)^{<r-1}$.

Lemma 4.12. The K-vector space

$$
L_{i}^{<r-1}:=\bigoplus_{m<r-1} L_{i}^{(m)}
$$


is a right $S$-module.

Proof. Suppose that $0 \leq m<r-1$. Let $\theta \in L_{i}^{(m)} \subseteq \mathscr{D}(I)$. For $f \in S$,

$$
\theta f(Q S)=\theta(Q f S) \subseteq I \text {. }
$$

Thus $\theta f \in \mathscr{D}(I)$. It follows from Proposition 2.1 that $\theta f \in \bigoplus_{\ell=0}^{m} \mathscr{D}^{(\ell)}(I)$. The operator $\theta f$ is divisible by the polynomial $p_{1} \cdots p_{i}$ since $\theta \in p_{1} \cdots p_{i} \mathscr{D}^{(m)}(S)$. Thus each homogeneous component of $\theta f$ is divisible by $p_{1} \cdots p_{i}$. It follows that

$$
\theta f \in \bigoplus_{\ell=0}^{m}\left(\mathscr{D}^{(\ell)}(I) \cap\left(p_{1} \cdots p_{i}\right) \mathscr{D}^{(\ell)}(S)\right)=\bigoplus_{\ell=0}^{m} L_{i}^{(\ell)} .
$$

Hence $L_{i}^{<r-1} \cdot S \subseteq L_{i}^{<r-1}$.

The following holds in general.

Proposition 4.13. As a vector space,

$$
\bigoplus_{|\alpha|<r-1} S \partial^{\alpha}=\bigoplus_{|\alpha|<r-1} \partial^{\alpha} S
$$

Define a right $S$-module $\mathscr{D}(S)^{<r-1}:=\bigoplus_{|\boldsymbol{\alpha}|<r-1} \partial^{\boldsymbol{\alpha}} S$. Then $\mathscr{D}(S)^{<r-1}$ is the module of differential operators of order less than $r-1$ by Proposition 4.13. By Lemma 4.12, we have the inclusion of right $S$-modules:

$$
L_{i}^{<r-1} \subseteq \mathscr{D}(S)^{<r-1} .
$$

Lemma 4.14. The right $S$-module $\left(L_{i-1} / L_{i}\right)^{<r-1}$ is Noetherian.

Proof. Since $\mathscr{D}(S)^{<r-1}$ is a finitely generated right $S$-module, $\mathscr{D}(S)^{<r-1}$ is Noetherian as a right $S$-module. Hence the subquotient $\left(L_{i-1} / L_{i}\right)^{<r-1}=\left(L_{i-1}^{<r-1}+L_{i} / L_{i}\right)$ of $\mathscr{D}(S)^{<r-1}$ is Noetherian as a right $S$-module.

Lemma 4.15. The right $\mathscr{D}(I)$-module $L_{i-1} / L_{i}$ is Noetherian.

Proof. By Corollary $4.11, N:=\left(L_{i-1} / L_{i}\right)^{\geq r-1} \cdot \mathscr{D}(I)$ is Noetherian as a right $\mathscr{D}(I)$ module. Consider the factor $N^{\prime}:=\left(L_{i-1} / L_{i}\right) / N$. It is clear that as a right $S$-module, $N^{\prime}$ is a factor of $\left(L_{i-1} / L_{i}\right)^{<r-1}$. Thus $N^{\prime}$ is Noetherian as a right $S$-module as so certainly as a right $\mathscr{D}(I)$-module. By [2, Proposition 1.2], $L_{i-1} / L_{i}$ is Noetherian as a right $\mathscr{D}(I)$-module.

Theorem 4.16. The ring $\mathscr{D}(S / I) \simeq \mathscr{D}(I) / I \mathscr{D}(S)$ is Noetherian (i.e., $\mathscr{D}(S / I)$ is right Noetherian and left Noetherian).

Proof. By Lemma 4.15 and by considering the sequence (4.2), we see that the ring $\mathscr{D}(I) / I \mathscr{D}(S)$ is right Noetherian. Therefore, by Corollary 3.3, we conclude that the ring $\mathscr{D}(S / I) \simeq \mathscr{D}(I) / I \mathscr{D}(S)$ is Noetherian. 
It is known that idealisers in the second Wely algebra may or may not be Noetherian ([9, Theorem 2]). However, the ideal $I$ dose not satisfy the hypothesis of $[9$, Theorem 2]. The Noetherian property of the idealiser $\mathscr{D}(I)$ is still open.

In the rest of this section, we give an example of a family of Noetherian rings whose graded rings associated to the order filtration are not Noetherian.

By Proposition 2.1, we can decompose $\mathscr{D}(I) / I \mathscr{D}(S)$ into the direct sum

$$
\mathscr{D}(I) / I \mathscr{D}(S)=\bigoplus_{m \geq 0}\left(\mathscr{D}^{(m)}(I) / I^{(m)}(S)\right)
$$

as a left $S$-module. The order filtration of $\mathscr{D}(I) / I \mathscr{D}(S)$ is the filtration $\mathcal{F}=$ $\left\{F_{m}\right\}_{m \geq 0}$ defined by

$$
F_{m}=\bigoplus_{\ell \leq m}\left(\mathscr{D}^{(\ell)}(I) / I^{(\ell)}(S)\right) .
$$

We denote by $S_{j}$ the $K$-vector subspace of $S$ spanned by the monomials of degree $j$. An element $\theta=\sum_{\alpha} f_{\boldsymbol{\alpha}} \partial^{\alpha} \in \mathscr{D}(S)$ is of polynomial degree $k$, if $k$ is the smallest integer such that $f_{\boldsymbol{\alpha}} \in \bigoplus_{j=0}^{k} S_{j}$ for all $\boldsymbol{\alpha}$ with nonzero $f_{\boldsymbol{\alpha}}$.

Example 4.17. Let $S=k[x, y]$ be the polynomial ring, and let $I$ be the ideal generated by the polynomial $Q=p_{1} \cdots p_{r}(r \geq 2)$ defining a central arrangement.

The graded ring $\operatorname{Gr} \mathscr{D}(S / I)$ associated to the order filtration is a commutative ring. Let $\bar{\theta}$ be the image of $\theta \in \mathscr{D}(S / I)$ in $\operatorname{Gr} \mathscr{D}(S / I)$. We consider the ideal $M:=\left\langle\overline{P_{1} \delta_{1}^{m}} \mid m \geq 1\right\rangle$ of $\operatorname{Gr} \mathscr{D}(S / I)$.

Assume that $M$ is finitely generated with generators $\eta_{1}, \ldots, \eta_{\ell}$. Then there exists a positive integer $m$ such that

$$
M=\left\langle\eta_{1}, \ldots, \eta_{\ell}\right\rangle \subseteq\left\langle\overline{P_{1} \delta_{1}}, \ldots, \overline{P_{1} \delta_{1}^{m-1}}\right\rangle .
$$

Since $\overline{P_{1} \delta_{1}^{m}} \in M$, we can write

$$
\overline{P_{1} \delta_{1}^{m}}=\overline{P_{1} \delta_{1}} \cdot \overline{\theta_{1}}+\cdots+\overline{P_{1} \delta_{1}^{m-1}} \cdot \overline{\theta_{m-1}}
$$

for some $\theta_{1}, \ldots, \theta_{m-1} \in \mathscr{D}(I)$.

If $\theta \in \mathscr{D}(I)$ with $\operatorname{ord}(\theta) \leq 1$, then the polynomial degree of $\theta$ is greater than or equal to 1 by Proposition 4.1. Since the order of the LHS of (4.11) equals m, there exists at least one $\theta_{j}$ such that the order of $\theta_{j}$ is greater than or equal to 1 . Thus the polynomial degree of the RHS of (4.11) is greater than $r-1$. However, the polynomial degree of the LHS of (4.11) is exactly $r-1$. This is a contradiction.

Therefore $M$ is not finitely generated, and thus we have proved that $\operatorname{Gr} \mathscr{D}(S / I)$ is not Noetherian.

\section{REFERENCES}

[1] Bernstein, J.N., Gel'fand, I.M., Gel'fand, S.I. (1972). Differential operators on the cubic cone. Russian Math. Surveys 27:169-174. 
[2] Goodearl, K. R., Warfield, R. B. (1989). An introduction to noncommutative noetherian rings. London Mathematical Society Student Texts, vol. 16 Cambridge University Press, Cambridge.

[3] Holm, P. (2002). Differential Operators on Arrangements of Hyperplanes. PhD. Thesis, Stockholm University.

[4] Holm, P. (2004). Differential Operators on Hyperplane Arrangements. Comm. Algebra 32:21772201.

[5] McConnell, J.C., Robson, J.C. (1987). Noncommutative Noetherian Rings. Pure and Applied Mathematics, John Wiley \& Sons, Chichester.

[6] Muhasky, J.L. (1988). The differential operator ring of an affine curve. Trans. Amer. Math. Soc. 307:705-723.

[7] Năstăsescu, C.,Van Oystaeyen, F. (1982) Graded ring theory. North-Holland, Amsterdam.

[8] Orlik, P.,Terao, H. (1992). Arrangements of Hyperplanes. Grundlehren dermatematischen Wissenschaften 300:Springer-Verlag.

[9] Resco R. (1984). Affine domains of fnite Gel'fand-Kirillov dimension which are right, but not left, Noetherian. Bull. London Math. Soc. 16:no. 6, 590-594.

[10] Saito, M., Takahashi, K. (2009). Noetherian properties of rings of differential operators of affine semigroup algebras. Osaka J. Math. 46:529-556.

[11] Smith, S.P., Stafford, J.T. (1988). Differential operators on an affine curve. Proc. London Math. Soc. (3) 56:229-259.

[12] Snellman, J. (2005). A Conjecture on Poincaré-Betti Series of Modules of Differential Operators on a Generic Hyperplane Arrangement. Experiment. Math. 14:445-456.

E-mail address: naka_n@math.sci.hokudai.ac.jp

Department of Mathematics, Graduate School of Science, Hokkaido University, SAPPORO, 060-0810, JAPAN 